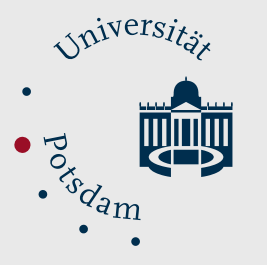

Philosophische Fakultät

Volkhard Wels

\title{
Rationalistische Begründung der Dichtung und Kritik des Enthusiasmus
}

Die Poetik Campanellas in ihren historischen Bezügen

Suggested citation referring to the original publication:

Scientia poetica : Jahrbuch für Geschichte der Literatur und der Wissenschaften 9 (2005)

ISSN 1431-5041

Postprint archived at the Institutional Repository of the Potsdam University in:

Postprints der Universität Potsdam

Philosophische Reihe ; 94

ISSN 1866-8380

http://nbn-resolving.de/urn:nbn:de:kobv:517-opus4-87221 

Rationalistische Begründung der Dichtung und Kritik des Enthusiasmus. Die Poetik Campanellas in ihren historischen Bezügen 

Volkhard Wels

\section{Rationalistische Begründung der Dichtung und Kritik des Enthusiasmus}

Die Poetik Campanellas in ihren historischen Bezügen 
Dieses Werk ist unter einem Creative Commons Lizenzvertrag lizenziert: Namensnennung - 4.0 International

Um die Bedingungen der Lizenz einzusehen, folgen Sie bitte dem Hyperlink:

http://creativecommons.org/licenses/by/4.0/

Universität Potsdam 2016

Online veröffentlicht auf dem

Publikationsserver der Universität Potsdam::

URN urn:nbn:de:kobv:517-opus4-87221

http://nbn-resolving.de/urn:nbn:de:kobv:517-opus4-87221 


\title{
Rationalistische Begründung der Dichtung und Kritik des Enthusiasmus
}

\author{
Die Poetik Campanellas in ihren historischen Bezügen
}

\begin{abstract}
The Poetica of Campanella is the expression of a strong rationalization and didactic instrumentalization of poetry. This rationalization can be explained as reaction to the theories of inspiration by Ficino and Pomponazzi, who both set the enthusiasm of the poet beside the revelation of the prophet and, by doing so, challenged the exclusive claim of prophetic revelation.
\end{abstract}

Tommaso Campanellas Poetik ist in mehreren Hinsichten richtungsweisend für die Dichtungsauffassung des 17. Jahrhunderts, und allein deswegen verdient sie in der Forschung weit mehr Aufmerksamkeit, als ihr bis jetzt zukam. ${ }^{1}$ Die Poetica steht, worauf schon die Tatsache hinweist, daß sie als vierter Teil von Campanellas Philosophia rationalis (1612 entstanden, 1638 gedruckt) ${ }^{2}$ erschienen ist, in einer rationalistischen Tradition, das heißt, sie begreift das dichterische Vermögen als eine technische Fähigkeit, die genauso wie die Fähigkeiten zu grammatisch korrektem, lo-

1 Zu Campanellas Poetik vgl. Lina Bolzoni: »La Poetica latina di Tommaso Campanella«, in: Giornale storico della letteratura italiana 149 (1972), S. 481-521; Noel L. Brann: The Debate over the Origin of Genius during the Italian Renaissance. The Theories of Supernatural Frenzy and Natural Melancholy in Accord and in Conflict on the Threshold of the Scientific Revolution. Leiden-Boston-Köln 2002 (Brill's Studies in Intellectual History 107), S. 412-441; Antonio Corsano: »La poetica del Campanella«, in: Giornale critico della filosofia italiana 39 (1960), S. 357-372, Winfried Schleiner: Melancholy, Genius and Utopia in the Renaissance. Wiesbaden 1991 (Wolfenbütteler Abhandlungen zur Renaissanceforschung 10) S. 45-51 und Daniel Pickering Walker: Spiritual and Demonic Magic from Ficino to Campanella. London 1958 (Studies of the Warburg Institute 22), S. 203-236.

2 Eine erste, italienische Fassung der Poetik ist bereits 1596 entstanden. Beide Fassungen wurden kritisch herausgegeben und kommentiert von Luigi Firpo, vgl. Tommaso Campanella: Poetica. Testo italiano inedito e rifacimento latino. A cura di Luigi Firpo. Roma 1944. (Reale accademia d'italia. Studi e documenti 13). Ich zitiere nach dieser Fassung. Eine Teilausgabe der lateinischen Fassung mit italienischer Übersetzung findet sich in Tommaso Campanella: Opere. A cura di Luigi Firpo. Milano 1954, S. 905-1219 und in Tommaso Campanella: Opere letterarie. A cura di Lina Bolzoni. Torino 1977, S. 457-663. 
gisch-argumentativem und rhetorisch gestaltetem Sprechen lehr- und lernbar ist. In diesem Sinne folgt die Dichtkunst innerhalb der Philosophia rationalis auf Grammatik, Dialektik und Rhetorik. Mit dieser Eingliederung der Poetik in die propädeutischen Fächer des universitären Triviums steht Campanella in einer langen Tradition, die auf die spätantike, arabische Rezeption des aristotelischen Organons zurückgeht und im lateinischen Kulturkreis von Thomas von Aquin über Girolamo Savonarola bis hin zu Iacopo Zabarella reicht. ${ }^{3}$

Dennoch ist Campanellas Poetik nicht mit der aristotelischen Tradition zu verrechnen, denn in den einzelnen Bestimmungen weicht sie erheblich von dieser ab. Campanella zelebriert diese Abweichungen förmlich mit Kapitelüberschriften, in denen >Aristoteles diametral entgegengesetzte Behauptungen angekündigt werden. Trotz dieser provokativen Formulierungen ist der eigentliche Gegner nicht Aristoteles, sondern, so meine zentrale These, die Inspirationstheorie des melancholisch begründeten Enthusiasmus, wie sie von Marsilio Ficino in einer neuplatonischen und von Pietro Pomponazzi in einer naturphilosophischen Deutung entwickelt wurde. Es handelt sich deshalb bei Campanellas Poetik um eine in ihren einzelnen Bestimmungen antiaristotelische Poetik, die aber auf einen rationalistischen - und damit grundsätzlich in der aristotelischen Tradition stehenden - Dichtungsbegriff zurückgreift, um die Dichtung ohne Rekurs

3 Ich kann auf diesen weiteren Kontext hier nur verweisen. Zur arabischen Rezeption des aristotelischen Organons vgl. die Literaturangaben unten Anm. 23. Thomas' Bestimmung der Poetik als Teil des Organons findet sich in seinem Kommentar zur Zweiten Analytik, vgl. Thomas von Aquin: »Expositio libri posteriorum«, in: ders.: Opera omnia. Iussu Leonis XIII P.M. edita. Cura et studio Fratrum Praedicatorum. Paris-Rom 1989, Bd. I.2, S. 3-9. Savonarolas Bestimmung der Poetik findet sich in seinem Apologeticus, vgl. Girolamo Savonarola: »Apologeticus de ratione poeticae artis«, in: ders.: Scritti filosofici. Edizione Nazionale delle Opere di Girolamo Savonarola. A cura di Giancarlo Garfagnini e Eugenio Garin. Roma 1982. Bd. 14.1, S. 209-272. Zu Thomas und Savonarola vgl. die entsprechenden Kapitel bei Concetta Carestia Greenfield: Humanist and Scholastic Poetics. 1250-1500. Lewisburg 1981. Iacopo Zabarellas Bestimmung der Poetik findet sich in seinem Traktat $\gg$ De natura logicae«, in: ders.: Opera Logica. Frankfurt a. M. 1608. Ndr. Frankfurt a. M. 1966. Zu Zabarella vgl. W. Edwards: "Jacopo Zabarella: A Renaissance Aristotelian's View of Rhetoric and Poetry and their Relation to Philosophy«, in: Arts Libéraux et Philosophie au Moyen Age. Actes du quatrième congrès international de philosophie médiévale (Montreal 1967). Montreal-Paris 1969, S. 843-854. Die damit angedeutete Tradition der Poetik als Teil des Organons war bisher, soweit ich sehe, nicht Gegenstand der Forschung. 
auf eine Inspirationstheorie zu begründen. Genau in diesen Punkten ist die Poetik Campanellas richtungsweisend für das 17. Jahrhundert.

Diese Interpretation der Poetik Campanellas entwickle ich in sechs Schritten. In einem ersten Schritt zeige ich die rationalistische Ausrichtung der Poetik in ihrer grundlegenden Bestimmung des >exemplum< als der logischen Form der Dichtung. Diese Bestimmung impliziert - zweitens - eine Kritik des aristotelischen Nachahmungsbegriffes. Dennoch kann man - drittens - nicht einfach sagen, Campanella stünde in einer antiaristotelischen Tradition, denn innerhalb der aristotelischen Tradition selbst muß zwischen einer aristotelischen und einer averroischen Richtung unterschieden werden. Campanella stellt sich bewußt in letztere Tradition.

Mit meinem vierten Punkt komme ich zum zweiten rationalistischen Merkmal der Poetik Campanellas, nämlich der physiologischen Bestimmung des Metrums als einer Affektation des >spiritus animalis<. >Schön $<$ wird nach Campanella das genannt, was den >spiritus animalis < (die vom Blut transportierten >Lebensgeister $<$ ) in seiner Konsistenz erhält. Durch diese physiologische Begründung kann Campanella die Dichtung zu einem Teil der >magia naturalis` erklären, das heißt zu einer Technik, die mittels in der Natur begründeter Ursachen natürliche Wirkungen hervorbringt. Diese physiologische Deutung des Metrums hat - fünftens - ihren eigentlichen Hintergrund in Campanellas Kritik des Enthusiasmus, das heißt in seiner Ablehnung jeder Form einer göttlichen Inspiration des Dichters. Mit dieser Kritik ist - sechstens - Campanella nur vor dem Hintergrund der divergenten Melancholie- und Enthusiasmus-Theorien Marsilio Ficinos, Gianfrancesco Picos und Pietro Pomponazzis zu verstehen. Gerade in der Abgrenzung von den Melancholie-Theorien von Ficino und Pomponazzi liegt das eigentliche Motiv für die rationalistische Ausrichtung von Campanellas Poetik.

\section{Die Definition der Dichtung: Poetik als Teil}

der $>$ philosophia rationalis $<$

Campanella definiert die Dichtkunst als »die technische Kunstfertigkeit eines sachverständigen Werkmeisters, mit dem Zweck, den Unwilligen oder Unfähigen das Wahre und Gute auf angenehme, leichte und unmerkliche Art einzuflößen ${ }^{4}{ }^{4}$ Als solche steht die Poetica an vierter Stelle in

4 Campanella: Poetica (wie Anm. 2), S. 219: »Poëticam esse sapientis architectonici artem instrumentalem ad propinandum iucunde, facile et inadvertenter verum bonum- 
Campanellas Philosophia rationalis, hinter Grammatik, Dialektik und Rhetorik und vor der Theorie der Geschichtsschreibung. Die Reihenfolge versteht sich im didaktischen Sinne, denn Grammatik, das heißt eine sprachlich korrekte Ausdrucksweise, Dialektik, das heißt die Fähigkeit zu logisch-argumentativem Sprechen, und Rhetorik, das heißt die Fähigkeit zu sprachlich gestaltetem Sprechen, werden für die Dichtung vorausgesetzt.

Darüber hinaus erklärt sich der Unterschied zwischen Dialektik, Rhetorik und Poetik durch die unterschiedlichen Anwendungsbereiche und die jeweilige Aufnahmebereitschaft der Zuhörer. Logisch-argumentatives Sprechen gehört nach Campanellas Überzeugung an die Schulen und Universitäten, wo die Zuhörer das Wahre suchen, rhetorisch gestaltetes Sprechen an öffentliche Orte wie Kirche und Ratsversammlung, an denen die Zuhörer dem Wahren geneigt sind, Dichtung aber an das Theater, an private Orte und überall dorthin, wo diejenigen sind, die das Wahre und Gute nicht hören wollen. Zweck der Dichtung sei es, die Zuhörer so zu täuschen, daß sie die unterschwellig im Vergnügen vermittelte Belehrung durch die Dichtung akzeptieren, gegen die sie sich ansonsten, würde sie in sachlich nüchterner Form vorgebracht, sträuben würden. ${ }^{5}$

Während der Redner immer ein spezifisches Gutes im Blick habe, also immer diesen Fürsten, diese Ratsversammlung oder diesen spezifischen Menschen anspreche, sei die Dichtung auf das allgemeine Gute ausgerichtet und betreffe deshalb alle Menschen. Sie stelle zwar einzelne Handlungen dar, indem sie dies aber in idealisierter Form tue, könne sie zum Vorbild oder abschreckenden Beispiel für alle werden. Die Rhetorik richte sich außerdem an Zuhörer, die zuhören wollen und über das dafür notwendige Mindestmaß an Bildung verfügen, die Dichtung aber an Zuhörer, die ungebildet und nicht fähig sind, der Belehrung in eigentlicher Form zu folgen.

Das Entscheidende an dieser Bestimmung der Dichtung ist offensichtlich das Vergnügen, das >delectare<, wie es mit der Horazischen Termino-

que nolentibus et incapacibus [...].« Übersetzungen hier und im Folgenden vom Verfasser

5 Ebd., S. 219: "Quemadmodum dialectica in scholis et ad philosophos verum audire volentes personat, rhetorica vero in templis et in foro ad senatum et populum et quicunque ad bona honesta utiliaque propensi sunt alloquitur, sic poëtica, in theatris et in privatis aedibus et ubique, verum et bonum audire nolentes ita mulcet et decipit, ut, dum voluptatis captandae gratia vel alterius negotii praetextu audiunt, veritatis bonitatisque praeceptione salutifera iocunde afficiuntur, quas, nisi sic propinarentur, illi aversarentur utique ex parte, vel prorsus.« 
logie heißt, durch das sich das >prodesse $<$, der didaktische Nutzen, vollzieht. Das Vergnügen der Dichtung besteht nach Campanella in zweierlei, nämlich im exemplarischen Charakter der Dichtung und in der metrischen Form der Darstellung. ${ }^{6}>$ Exemplarischer Charakter $<$ heißt im fachterminologischen, das heißt dialektischen Sinne, daß der Gegenstand der Dichtung die logische Form des >exemplum< hat, also einen Schluß von einem Einzelfall auf einen anderen Einzelfall darstellt. »Die ganze Dichtung ist ein Argument aus dem exemplum« heißt es kurz und bündig in Campanellas Dialectica. ${ }^{7}$ Im Gegensatz zum Syllogismus, der immer deduktiv eine besondere Aussage einer allgemeinen Aussage subsumiert - alle Menschen sind sterblich, Sokrates ist ein Mensch, also ist Sokrates sterblich -, hat das >exemplum « nur eine bedingte Gültigkeit im logischen Sinne, was aber an seiner rhetorischen Überzeugungskraft nichts ändert. Die Dichtung führt an einem Einzelschicksal vor, wohin dieses oder jenes lasterhafte Verhalten führt und erlaubt dadurch dem Zuhörer die Schlußfolgerung, daß dieses spezifische lasterhafte Verhalten bei ihm zum selben Erfolg führen wird: Diese Figur des Dramas ist für ihr lasterhaftes Verhalten bestraft worden, also werde auch ich bestraft werden, wenn ich mich ähnlich verhalte.

Die Anwendung der logischen Form des >exemplum dem intellektuellen Niveau der Leser oder Zuschauer, die einer rein logischen, das heißt sachlich nüchternen Darstellung nicht folgen können oder wollen. Oft mißfalle es den Zuhörern, so Campanella, auf ihre Fehler hingewiesen $\mathrm{zu}$ werden oder es langweile sie, sich moralische Belehrungen anzuhören; deswegen verwende der Dichter - neben der metrischen Form - >Umwandlungen $<$ oder figürliche Ausdrucksweisen (>transfigurationes sermonis $<$ ), »in denen er das eine sagt und etwas anderes bezeichnet, und das Schwerverständliche durch das Leichtverständliche erläutert. ${ }^{8}$

Metapher, Allegorie, Gleichnis (>parabola $<$ ) und $>$ fabula $<{ }^{9}$ haben als solche figürlichen Ausdrucksweisen denselben Ursprung, indem sie alle

\footnotetext{
Vgl. ebd., S. 220.

7 Tommaso Campanella: »Dialectica«, in: ders.: Philosophia rationalis, pars secunda. Paris 1638, S. 394: »Tota poëtica est argumentum ab exemplo.« Die Definition des sexemplum< lautet dort: »Exemplum est aliquid ostendere de uno singulari per aliud singulare, in quo idem praedicatum inest.«

8 Campanella: Poetica (wie Anm. 2), S. 239: »Propterea poëta, non contentus carmine, addidit transfigurationem sermonis, in quo aliud dicit et aliud significat et per clara obscura patefacit.«

9 Der Begriff der >fabula< ist aufgrund seiner Doppeldeutigkeit nicht zu übersetzen. Er bezeichnet einerseits als Übersetzung des aristotelischen >mythos< die Handlung eines
} 
nacheinander aus der Ähnlichkeit der Sachverhalte (>a similitudine rerum $<$ ) entstanden sind: die >fabula $<$ aus dem Gleichnis, das Gleichnis aus der Allegorie, die Allegorie aus der Metapher und die Metapher aus der Ähnlichkeit. ${ }^{10}$ Der Unterschied zwischen diesen vier Formen besteht darin, daß Metapher und Allegorie sich auf rein sprachlicher Ebene bewegen und eines (bei der Metapher) oder mehrere Worte (bei der Allegorie) entsprechend der Ähnlichkeit austauschen, während es bei Gleichnis und >fabula die Sachverhalte selbst sind, die etwas anderes bedeuten. Der Unterschied zwischen Gleichnis und >fabula besteht darin, daß das Gleichnis, wie es sich etwa im Neuen Testament findet, ausschließlich auf die ihm zugrundeliegende Lehre verweist, in der >fabula < dagegen für sich bedeutsame Personen und Ereignisse zum >exemplum < für unsere eigenen Verhaltensweisen werden:

Beachte, daß man bei einer fabula nicht etwas anderes als das Gesagte verstanden haben will, sondern daß dieses als exemplum für andere Sachverhalte und Personen dient, unter denen unsere Tugenden und Laster und was wir meiden und was wir suchen sollten, zu verstehen sind. ${ }^{11}$

In diesem Sinne beruhen die Komödien und Tragödien auf einer >fabula<. Die Tragödie zeige mit den Schicksalsschlägen und dem Tod der Fürsten, daß diese nicht auf ihr Glück vertrauen dürften, die Komödie zeige, wie sich die Menschen als solche verhielten.

Der Dichter ist nicht der einzige, der sich des sexemplum< bedient. Auch die Rhetorik tut es und die dialogische Prosa, genauso wie die Gesetzgebung, die an exemplarischen Fällen klar macht, was erlaubt ist und was nicht, genauso wie jede Fachschriftstellerei, etwa die Medizin. ${ }^{12}$ Was den spezifisch dichterischen Gebrauch von >exemplum< und >fabula< ausmacht, abgesehen von der metrischen Form, ist deren ideale Gestaltung. Die aristotelische Unterscheidung von Dichtung und Geschichtsschreibung im neunten Kapitel der Poetik (1451a) - nicht Vers und Prosa unterscheiden sie, sondern das Tatsächliche, historisch Besondere als Ge-

Dramas oder Epos, andererseits aber auch die Fabel im Sinne der Tierfabel, deren Verwandtschaft zum Gleichnis offensichtlich ist. Nicht zuletzt durch die Identifikation dieser beiden Begriffe von >fabula< dürfte die Frühe Neuzeit der Überzeugung gewesen sein, daß Aristoteles in der Poetik mit der >fabula die Gleichnishaftigkeit für das Wesen der Dichtung hält.

10 Vgl. Campanella: Poetica (wie Anm. 2), S. 264f.

11 Ebd., S. 241: „Nota, quod in fabulis nolumus aliud quam dicimus intelligi, sed ponimus illas ut exempla in aliis rebus et personis, in quibus nostras virtutes vel vitia, et quid cavendum, quid sectandum intelligimus."

12 Vgl. ebd., S. 257. 
genstand der Geschichtsschreibung und das Mögliche, mit einer höheren, allgemeineren Bedeutung als Gegenstand der Dichtung - interpretiert Campanella in dem Sinne, daß nicht der Vers allein das Wesen der Dichtung ausmacht, sondern auch der Stoff der Dichtung einen spezifisch exemplarischen Charakter haben und damit Ausdruck eines >herausragenden Vorbilds $<$ ( $>$ idea excellens $<$ ) sein muß.

Als Ausdruck einer >idea< ist das >exemplum< in seinem Sein etwas Besonderes, in seiner darstellenden und mahnenden Absicht aber etwas Allgemeines. Dichter und Historiker haben also beide das Besondere als Stoff, nur daß es vom Historiker als Sachverhalt beschrieben wird, vom Dichter aber als >exemplum $<$ : »Das exemplum aber ist ein einziges in seinem Sein und ein Allgemeines in seinem Darstellen. « ${ }^{13}$ Wo der >historia< - als dem Gegenstand der Geschichtsschreibung - der herausragende Charakter fehlt, muß sich der Dichter einer >fabula< bedienen und der >historia< dadurch exemplarischen Charakter verleihen. Dieser Charakter fehlt dem Geschichtswerk Herodots. Auch in Verse gebracht, ist dieser Inhalt einer Dichtung nicht würdig.

\section{Kritik des aristotelischen Nachahmungsbegriffes}

Definierendes Merkmal der Dichtung ist nach Campanella aber nicht das >exemplum<, sondern das Metrum:

Das eigentümliche Merkmal [der Dichtung] jedoch, durch das sie sich von allen übrigen Künsten unterscheidet, ist das Metrum, das die angenehmste Art des Sprechens ist, der Seele eingeboren. Sie reißt diese auf wundersame Art mit sich und affiziert sie mit der Überzeugung, die sie mit sich führt. ${ }^{14}$

Diese Definition der Dichtung über das Metrum ist explizit gegen die aristotelische Definition der Dichtung als Nachahmung (>mimesis $<$, >imitatio<) einer Handlung (Poetik 1447a) gerichtet. Der Vorteil von Campa-

13 Die Passage lautet in ihrem Kontext, Campanella: Poetica (wie Anm. 2), S. 258: »Et quia exemplum idea est, licet singularis, tamen in repraesentando et monendo est universalis: idcirco poëta versatur circa universale. Nec differt ab historico, ut putat Aristoteles, propter hoc, quod historicus singulare tractat: nam illud idem singulare est utriusque interdum materia, sed quia ab historico describitur ut res, a poëta adaptatur ut exemplum. Exemplum autem unum est in essendo, universaleque in repraesentando."

14 Ebd., S. 220: »Proprium tamen, quo a cunctis artificibus differt, metrum habet, qui suavissimus est dicendi modus innatus animae, ipsamque rapiens mirifice, afficiensque sententia, quam secum fert.« 
nellas Definition der Dichtung über das Metrum besteht darin, daß Campanella den lyrischen Formen wie Elegie, Hymne und Psalm, genauso wie den Lehrgedichten (Empedokles, Lukrez), denen Aristoteles aufgrund mangelnder Handlung den Namen der Dichtung verweigern muß, Gerechtigkeit widerfahren lassen kann. Auf der anderen Seite ist Campanella seinerseits gezwungen, allen literarischen Prosaformen den Namen der Dichtung zu bestreiten. ${ }^{15}$

Würde die Handlung das Wesen der Dichtung bestimmen, wären, so Campanella, Ovid mit seiner Liebeskunst und Vergil mit seinen Georgica keine Dichter, wohl aber mit den Metamorphosen und der Aeneis. Orpheus, Musaeus, Horaz und Petrarca könnten keine Dichter heißen, statt dessen wären Platon, Boccaccio und Äsop Dichter, obwohl sie, wie Campanella schreibt, nur Dialoge geschrieben und Personen nachgeahmt hätten. Die christlichen Hymnen wären nur das Werk von Versifikatoren, während die Amadis- und Artus-Romane und ähnlich schädliche Lügengeschichten das Werk von Dichtern wären. Absurd wäre es, zu behaupten, Lukan wäre ein Dichter, wenn er die Taten Cäsars erfunden hätte, aber er sei kein Dichter, weil sie wahr sind. ${ }^{16}$

Die Nachahmung als solche kann auch nicht den Zweck der Dichtung darstellen, sondern nur das Mittel, dessen sich die Dichtung bedient, um ihren Lehrgehalt zu vermitteln:

Ziel und Kunst des Dichters ist es deshalb nicht, nachzuahmen und zu erdichten, sondern er ahmt nach, um darzustellen, er stellt dar, um emotional anzusprechen und zu lehren, er lehrt und spricht emotional an, um von der Einhaltung der Gesetze, der Tugend und einem glückseligen Leben zu überzeugen. ${ }^{17}$

Was den Namen der Dichtung verdienen soll, muß moralisch und pädagogisch wertvoll sein, das heißt die Tugend loben und das Laster tadeln.

Mit diesem Argument bestreitet Campanella, daß Homer, Catull und Martial Dichter sind, denn sie sind dem Gemeinwesen schädlich, dringen nicht auf die Einhaltung der Gesetze und fordern nicht zu tugendhaftem Verhalten auf. Vergil ist wegen der schamlosen Dido-Geschichte allen Tadels würdig, nur seine Georgica sind es wert, erhalten zu werden. Wenn schon Platon die Dichter aus seinem Staat verbannt hat, um wieviel mehr steht es den Christen an, solche Dichter aus ihrem Reich zu verban-

15 Vgl. ebd., S. 244f.

16 Vgl. ebd., S. 247-250.

17 Ebd., S. 244: »Poëtae igitur finis et ars non est imitari et fingere, sed imitatur ut repraesentet, repraesentat ut afficiat et doceat, docet et afficit ut suadeat legem, virtutes et beatam vitam.« 
nen. Völlig zu Recht führe der Papst deshalb einen Index der verbotenen Bücher, auf den diese Werke zusammen mit denen der Ketzer gehörten. ${ }^{18}$

Gegen die aristotelische Bestimmung der Dichtung über die Nachahmung einer Handlung spricht außerdem das Argument, daß es eine Dichtung gibt, die nicht auf Handlung beruht, etwa wenn der Dichter sich mit der bloßen Beschreibung von Engeln und Teufeln begnüge oder die Tugenden und Laster in allegorischer Form als körperliche Wesen darstelle. ${ }^{19}$ Eine solche bloß beschreibende Dichtung müsse in der Definition der Dichtung mit erfaßt werden.

Dazu kommt drittens das Argument, daß die Nachahmung einer Handlung als Legitimation der Erfindung dienen kann, und das heißt als Legitimation der Lüge: »Wenn die aristotelischen Begründungen gültig wären, hätten wir keine Dichter, sondern nur Lügner, und die Dichtung wäre eine Tochter des Teufels, der der Vater der Lüge ist. « ${ }^{20}$ Zwar revidiert Campanella später die Bezeichnung der Dichtung als Lüge, indem der gleichnishafte Charakter der >fabula $<$ nicht darauf ausgerichtet sei zu täuschen, wie es der Lüge entspricht, sondern der Wahrheit diene. Damit ist jedoch nicht die Erfindung von Fabelwesen wie Chimären und Zentauren legitimiert. Der Dichter darf sich der >fabula<, der erfundenen Handlung, nur bedienen, wo kein wahres >exemplum < zur Verfügung steht, und wenn er eine Handlung erfindet, dann darf diese nur dazu dienen, die ihr zugrundeliegende Wahrheit oder Lehre gleichnishaft zu vergegenwärtigen. Eine Handlung, die nicht gleichnishaft, das heißt exemplarisch für einen bestimmten Lehrgehalt wäre, ist nicht zu legitimieren. ${ }^{21}$

\section{Campanella innerhalb der aristotelischen und averroischen Tradition}

Mit diesen Bestimmungen grenzt Campanella sich scharf von der aristotelischen Poetik ab, wobei an dieser Tatsache nicht nur interessant ist, wie genau Campanella die aristotelische Poetik kennt. Dies ist keine Selbstverständlichkeit, denn um 1600 ist die aristotelische Poetik gerade erst seit hundert Jahren überhaupt bekannt, ihre inhaltliche Erschließung hat erst um 1550 mit den Kommentaren von Lombardi, Maggi und Robortel-

\footnotetext{
18 Vgl. ebd., S. 254 und 259f.

19 Vgl. ebd., S. 256.

20 Ebd., S. 247: „Si Aristotelis rationes valent, poëtas non habemus, nisi mendaces, eritque poësis filia diaboli, qui est pater mendacii.«

${ }^{21}$ Vgl. ebd., S. 259.
} 
lo begonnen, erst um 1570 schließlich hat sich die aristotelische Poetik mit den Kommentaren von Vettori, Castelvetro und Riccoboni fest im universitären Kanon etabliert. ${ }^{22}$ Aus diesen Kommentaren bezieht Campanella seine Kenntnis der aristotelischen Poetik, im Gegensatz zu diesen Kommentaren aber steht seine scharfe Kritik an den aristotelischen Bestimmungen.

Damit ist jedoch nicht gesagt, daß Campanella mit seinen poetologischen Bestimmungen keine Vorgänger gehabt hätte, im Gegenteil. Campanella steht in einer weit zurückreichenden Tradition, die sich ebenfalls auf die aristotelische Poetik beruft, allerdings nicht auf ihre griechische Fassung, sondern auf die arabische. Um 1175 hat Averroes die aristotelische Poetik für die arabische Kultur des 12. Jahrhunderts reformuliert, und im Gegensatz zur lateinischen Übersetzung des griechischen Textes, deren Rezeption sich kaum nachweisen läßt, ist die Poetik des Averroes in mehreren lateinischen Übersetzungen bis weit ins 16. Jahrhundert hinein gedruckt worden, wobei sie ihre Autorität wesentlich aus der Tatsache bezog, daß sie mit der aristotelischen Poetik identifiziert wurde. ${ }^{23}$ Es wäre

22 Zur Rezeption der aristotelischen Poetik vgl. Renato Barilli: »La letteratura e la lingua, le poetiche e la critica d'arte, §100-109«, in: Il Cinquecento. Dal rinascimento alla controriforma. Roma 1973 (La letteratura italiana. Storia e testi 4.2), S. 506560; Renato Barilli: Poetica e retorica. 2. Aufl. Milano 1984 (Saggi di Estetica e di Poetica 9); Baxter Hathaway: The Age of Criticism. The Late Renaissance in Italy. Ithaca-N.Y. 1962; Rainer Stillers: Humanistische Deutung. Studien zu Kommentar und Literaturtheorie in der italienischen Renaissance. Düsseldorf 1988 (Studia humaniora 11); E. N. Tigerstedt: »Observations on the Reception of the Aristotelian Poetics in the Latin West «, in: Studies in the Renaissance 15 (1968), S. 7-24; Bernhard Weinberg: A History of Literary Criticism in the Italian Renaissance. 2 Bde. Chicago 1961.

Zur arabischen Fassung und ihrer lateinischen Rezeption verweise ich stellvertretend auf Judson Boyce Allen: »Hermann the German's Averroistic Aristotle and Medieval Poetic Theory«, in: Mosaic. A Journal for the Comparative Study of Literature and Ideas 9 (1976), S. 67-81. Später wiederaufgenommen in ders.: The Ethical Poetic of the Later Middle Ages. A Decorum of Convenient Distinction. Toronto 1982; Osborn Bennett Hardison: »The Place of Averroes' Commentary on the Poetics in the History of Medieval Criticism«, in: Medieval and Renaissance Studies 4 (1970), S. 57-81. Gekürzt wiederholt in: Classical and Medieval Literary Criticism. Translations and Interpretations, hg. v. A. Preminger, O. B. Hardison, Jr. und K. Kerrane. New York 1974, S. 341-348; Tigerstedt: Observations on the Reception of the Aristotelian Poetics (wie Anm. 22); Weinberg: A History of Literary Criticism (wie Anm. 22) sowie die Einleitung von A. J. Minnis and A. B. Scott in: Medieval Literary Theory and Criticism, c. 1100-c. 1375. The Commentary Tradition, hg. v. A. J. Minnis and A. B. 
zu zeigen, wie sich in den Poetik-Kommentaren des 16. Jahrhunderts die Erschließung des griechischen Textes nur mühsam von den Begriffen der arabischen Fassung befreit, denn, und das ist der entscheidende Punkt, die Poetik des Averroes weicht in den grundsätzlichen Bestimmungen erheblich vom aristotelischen Text ab. ${ }^{24}$

So bestimmt Averroes das Lob des tugendhaften und den Tadel des lasterhaften Verhaltens als den Zweck der Dichtung, das heißt, er stellt die Dichtung in den Dienst einer didaktischen Vermittlung der Moralphilosophie. Ihr didaktisches Ziel verfolgt die Dichtung durch ihren wesentlich gleichnishaften Charakter, wobei Averroes zwar nicht, wie später Savonarola und Zabarella, das >exemplum< zur logischen Form der Dichtung erklärt, aber den grundsätzlich argumentativen Charakter der Dichtung ganz analog betont. Den aristotelischen Nachahmungsbegriff deutet Averroes als Gleichnishaftigkeit, und wie bei Campanella heißt es außerdem, daß diese primitive argumentative Form der Dichtung sich besonders an das einfache Volk richte, das komplexeren argumentativen Verfahren nicht zugänglich sei. Ebenfalls wie Campanella bestimmt Averroes die metrische Form der Darstellung als definierendes Merkmal der Dichtung und - letzter Punkt - legt die Dichtung auf die Darstellung des Tatsächlichen fest, das heißt, er verbietet jede Art der >lügnerischen Erfindung< zum Zweck der bloßen Unterhaltung.

Offensichtlich schließt Campanella sich in allen entscheidenden Bestimmungen seiner Poetik dieser arabischen Tradition an, wenn auch ohne dies explizit zu vermerken. Während die Kommentatoren der aristotelischen Poetik sich in der zweiten Hälfte des 16. Jahrhunderts zu der Einsicht durchringen, daß die arabische Fassung keine Hilfestellung bei der Erschließung des griechischen Textes darstellt, und sich deshalb zuse-

Scott with the Assistance of David Wallace. Revised Edition. Oxford 1991, S. 277288.

Ich kann auf die verschiedenen lateinischen Fassungen hier nicht näher eingehen und nur grob auf die Quellen verweisen. Die lateinische Übersetzung des arabischen Textes von Hermannus Alemannus findet sich unter dem Titel »Expositio media Averrois sive Poetria Hermanno Alemanno interprete«, in: Aristoteles latinus, hg. v. Laurentius Minio-Paluello. Leiden 1968, Bd. 33, S. 40-74. Diese lateinische Fassung wurde wiederum zweimal ins Englische übersetzt. Eine vollständige, aber mit Vorsicht zu gebrauchende Übersetzung von Hardison findet sich in: Classical and Medieval Literary Criticism (wie Anm. 23), S. 349-382; eine Teilübersetzung mit wertvollem Kommentar von Minnis und Scott findet sich in: Medieval Literary Theory and Criticism (wie Anm. 23), S. 289-307. Die Übersetzung von Iacobo Mantino wurde nach der Ausgabe Venedig: Iunctas 1562 herausgegeben von Friedrich Heidenhain in: Jahrbücher für classische Philologie, Supplementband 17 (1890), S. 351-382. 
hends von dieser abwenden, kommt Campanella zu der genau gegenteiligen Schlußfolgerung, nämlich zu der Entscheidung, sich von der aristotelischen Poetik abzuwenden. Campanella erkennt, daß sich die arabische Tradition einer didaktischen, logisch verfahrenden Poetik nicht mit der griechischen verrechnen läßt, und entscheidet sich nicht für die griechische, sondern für die arabische Tradition.

Diese Bezüge, die hier mehr behauptet als bewiesen werden, müßten durch Detailstudien erhärtet werden, die jedoch ihrerseits eine genaue Darstellung der arabischen Fassung der aristotelischen Poetik voraussetzen würden. Dies kann hier nicht geleistet werden. Ich begnüge mich deshalb mit diesem Hinweis auf die arabische Tradition und kehre zur Definition der Dichtung in der Poetik Campanellas zurück, deren zweites, wesentliches Element, neben dem >exemplum<, die physiologische Wirkung des Metrums ist.

\section{Dichtung als Teil der >magia naturalis $<$}

Neben dem exemplarischen Gehalt ist die metrische Form der Darstellung für das Vergnügen verantwortlich, das die Dichtung verschafft, und damit für die Tatsache, daß der didaktische, im >exemplum< formulierte Gehalt der Dichtung den Leser erreicht. Auch hier grenzt sich Campanella scharf von Aristoteles ab. Die metrische Form mußte nämlich nicht erst erfunden werden, wie Aristoteles glaubt, sondern ist den Menschen angeboren, genauso wie den Vögeln ihr Gesang. Campanella belegt dies unter anderem mit einem Verweis auf die Bauern, die aus mnemotechnischen Gründen wichtige Ereignisse in metrischer Form überliefern, auf Sprichworte, die aus demselben Grund oft metrische Formen haben, und mit einem Verweis auf die neu entdeckten Naturvölker Amerikas, die sich ebenfalls metrischer Formen bedienen. ${ }^{25}$

Der Angeborenheit des Metrums entspricht seine physiologische Wirkung, das heißt die Affektion des >spiritus animalis $<$ :

Das behaupte ich in der Tat, daß das metrisch abgefaßte Lied keine andere Kraft hat als die, den spiritus animalis zu erfreuen, wie ich oben gesagt habe, und dadurch den Geist mit Vergnügen zu affizieren. ${ }^{26}$

\footnotetext{
25 Vgl. Campanella: Poetica (wie Anm. 2), S. 262f.

26 Ebd., S. 242: »Hoc quidem affirmo, carmen ut metricum non habere vim, nisi mulcendi spiritum animalem, uti supra diximus, ac per hoc mentem afficere voluptate $[\ldots] . \ll$
} 
Damit verweist Campanella auf das vorhergehende, zweite Kapitel seiner Poetik, in dem sich eine detaillierte Beschreibung der physiologischen Wirkung von Musik und Dichtung findet. Campanella führt in diesem Kapitel den Beweis, daß Vergnügen und Unterhaltung (>voluptas $<$ ) nicht der Zweck der Dichtung sind, sondern nur das Mittel, dessen die Dichtung sich bedient, um ihren Zweck - Belehrung im weitesten Sinne - zu erreichen.

$$
\text { »Vergnügen«, heißt es dort im Titel des zweiten Articulus, }
$$

ist die Stimmung, die der Empfindung eines auf uns bezogenen Gutes folgt. Ein Gut ist das, was uns durch irgendeine Erhaltung erhält, und deswegen bringt uns die [künstlerische] Nachahmung Vergnügen, denn sie ist der Anfang und die Grundlage der Kunstfertigkeit, durch welche sich das menschliche Geschlecht erhält. [...] Die Melodie aber und das Metrum sind nicht erfreulich, weil sie Nachahmungen sind, wie jener [scil. Aristoteles] sagt, sondern weil sie unmittelbar den spiritus in seinem Wesen und seiner Funktion erhalten, nicht aber weil sie Zeichen oder der Anfang von einem Gut sind, auch nicht akzidentiell. ${ }^{27}$

Gut ist und Vergnügen bereitet, was den Menschen in seinem Zustand erhält, und in der Folge des Kapitels definiert Campanella Schönheit als das Zeichen einer solchen Erhaltung: »Das aber, was die Empfindung der Erhaltung wie in einem Zeichen enthält, wird schön genannt $\ll .{ }^{28}$ Dabei sei es nicht das Nachgeahmte, was die Empfindung des Vergnügens auslöse, sondern die Qualität der Nachahmung. Ein von Michelangelo gemalter Affe sei schöner - und damit >vergnüglicher< - als die schönste Frau, auf unfähige Weise dargestellt. Die Hölle Dantes sei schöner als sein Paradies, denn die Personen, die Dante im »Inferno« beschreibt, seien besser getroffen als die im »Paradiso« dargestellten.

Das Vergnügen an Melodie und Metrum erklärt sich aus derselben Wurzel, denn auch diese geben uns die Empfindung der >Erhaltung $<$.

In uns ist nämlich ein warmer, feiner, beweglicher und klarer spiritus, der sich in den Höhlungen des Gehirns aufhält und sich von dort über die Nerven verteilt, dadurch den ganzen Körper in den Zustand der Empfindung und Bewegung versetzt und die Tätigkeiten der Empfindung, Bewegung und Ernährung ausübt. [...]

27 Ebd., S. 226: »Voluptatem esse affectionem sequentem sensum boni coniuncti nobis. Bonum esse id, quod nos conservat quacunque conservatione: et imitationem propterea esse voluptuosam, quoniam est artis, qua servatur humanum genus, exordium et constitutio. [...] Melodiam vero ac metrum non esse iocunda quia sunt imitationes, ut ille ait, sed quia spiritum in suo esse et ratione servant immediate, non quia signa aut principium bonorum, nisi per accidens.«

28 Ebd., S. 226: »Quae vero habent sensum conservationis sicuti in signo, dicuntur pulcra $[\ldots] . \lll$ 
und weil dieser spiritus von beweglicher Natur ist, freut er sich über Bewegung, denn er wird zu der Tätigkeit angeregt, die ihm wesentlich ist, nämlich der Bewegung. So wird er in Schwingung versetzt, vergrößert, gestärkt und von gefährlichem Ruß [wie ihn die schwarze Galle der Melancholie erzeugt, darauf wird zurückzukommen sein] gereinigt. Der Klang nun und der Gesang sind Bewegungen, die durch die Luft, den allgemeinen spiritus, zum Trommelfell im Mittelpunkt der Ohren getragen werden, wo der aus dem Gehirn kommende spiritus sich von den äußeren Bewegungen erregt fühlt und durch die Bewegungen eben das Bewegliche erkennt. ${ }^{29}$

Das heißt, der >spiritus` unterscheidet hier die verschiedenen Laute, Stimmen und Klänge der Musikinstrumente voneinander.

Das physiologische Modell, das dieser Vorstellung zugrundeliegt, geht auf Galen zurück. ${ }^{30}$ Der >spiritus animalis $<$ ist der >Lebensgeist $<$, der nach der medizinischen Vorstellung der Zeit im Gehirn durch eine Verfeinerung des mit dem Blut transportierten >spiritus vitalis< entsteht. In den Gehirnventrikeln ist der in den Nerven transportierte >spiritus animalis< das Medium, dessen sich die Gedanken und sinnlichen Wahrnehmungen bedienen. Durch die Sinneseindrücke wird der >spiritus animalis in Schwingungen versetzt und im Gehirn so verteilt und angeordnet, daß Bilder oder Eindrücke der wahrgenommenen Objekte entstehen. Wird ein Bild im Gedächtnis gespeichert, drücken die >spiritus < dieses Bild im hintersten Ventrikel des Gehirns, in dem die >memoria< lokalisiert wurde, wie in Wachs ab, von wo aus es dann vom >intellectus $<$ jederzeit wieder evoziert werden kann, indem die >spiritus< wieder die Form der entsprechenden Wahrnehmung annehmen. Entscheidende Bedeutung hat der >spiritus animalis < für die Entstehung der Affekte, denn ein Affekt oder

${ }^{29}$ Ebd., S. 228: »Est quidem in nobis spiritus calidus, subtilis, mobilis, lucidus, habitans in cerebri cavitatibus, inde et per nervos se diffundens, totum corpus sensificans et movens, et opera sensus et motus et nutritionis exercens. [...] cumque iste spiritus sit natura mobilis, gaudet motu, quoniam invitatur ad operationem sui nativam, quae est motus: item ventilatur, amplificatur, roboratur et fuliginibus infestantibus purgatur. Sonus autem et cantus motus sunt, per aerem, spiritum communem, delati in tympanum in centro aurium, ubi spiritus, e cerebro exiens, se agitari ab exterioribus motibus sentit ac proinde per motus ipsa mobilia cognoscit [...].«

30 Stellvertretend zur Spirituslehre verweise ich auf Jürgen Helm: »Die >spiritus< in der medizinischen Tradition und in Melanchthons Liber de anima«, in: Melanchthon und die Naturwissenschaften seiner Zeit, hg. v. Günther Frank u. Stefan Rhein. Sigmaringen 1998 (Melanchthon-Schriften der Stadt Bretten 4), S. 219-237 und Gerhard Klier: Die drei Geister des Menschen. Die sogenannte Spirituslehre in der Physiologie der Frühen Neuzeit. Stuttgart 2002 (Sudhoffs Archiv. Beihefte 50), beide mit weiterführender Literatur. 
Gefühl entsteht, indem die affektauslösende Wahrnehmung vom Gehirn über die >spiritus< zum Herzen als dem Sitz des Gefühls weitergeleitet wird. Das Herz reagiert auf die >spiritus « seinerseits mit einer Bewegung, die über den >spiritus vitalis $<$ auf den Körper übertragen und als emotionale Reaktion wahrgenommen wird.

$\mathrm{Zu}$ laute, scharfe und unregelmäßige Klänge, wie sie etwa der Krieg erzeugt, zerstören die >spiritus< und werden deshalb als unangenehm empfunden, mäßig laute und melodische Klänge dagegen unterstützen durch die dem Rhythmus und Metrum zugrundeliegende Ordnung den Erhaltungszustand der >spiritus< und werden als angenehm wahrgenommen. Campanella zufolge sind Metrum und Takt geradezu aus der Bewegung der >spiritus $<$, wie sie sich im Pulsschlag manifestiert, abgeleitet. Aus der unterschiedlichen Qualität der >spiritus « und ihrem jeweiligen Mischungsverhältnis erklärt sich die Tatsache, daß jeder eine andere Art von Musik als schön empfindet und die individuelle Wahrnehmung der Musik von dem jeweils aktuellen Mischungsverhältnis der >spiritus< abhängt. Aus diesem Abhängigkeitsverhältnis erklärt sich für Campanella die Tatsache, daß Musik sowohl das Verhalten der Menschen beeinflussen wie auch Krankheiten heilen kann. ${ }^{31}$

Wenn es also bei Campanella heißt, daß das Metrum die Kraft hat, den >spiritus animalis $<$ zu erfreuen und dadurch den Geist mit Vergnügen zu affizieren, ist dies in dem skizzierten physiologischen Sinne zu verstehen. Das Metrum oder die Melodie wirkt, durch den >spiritus animalis $<$, unmittelbar auf den Gemütszustand des Menschen ein, ohne daß der Intellekt Einfluß darauf nehmen könnte. Über den >spiritus< wirkt die Musik auf die Affekte ein, und die Affekte beeinflussen unmittelbar den Willen des Menschen, ohne daß diese Beeinflussung von Intellekt und >ratio< kontrolliert werden könnte. Der Mensch kann, durch Metrum und Melodie, unmittelbar zu einer konkreten Handlung oder einer allgemeinen Verhaltensweise gebracht werden, und das ist der Grund, warum die Dichtung, und die Musik, Teile der >magia naturalis « sind. Durch die Anwendung natürlicher Mittel - im Gegensatz zur Beschwörung von Engeln oder Dämonen in der göttlichen oder dämonischen Magie - wird eine natürliche Wirkung hervorgebracht. Durch sie gelingt es der Dichtung, »diejenigen, die nicht hören wollen, dazu zu bringen, zuzuhören und diejenigen

31 Vgl. Campanella: Poetica (wie Anm. 2), S. 229. 
zu belehren, die wegen ihrer Unerfahrenheit dazu nicht imstande sind oder von der Langeweile abgeschreckt werden «. ${ }^{32}$

Durch das Metrum steht die Dichtung in engstem Verhältnis zur Musik, von der sie neben dem Metrum auch noch anderes übernehmen kann, wie den Gesang und die instrumentale Begleitung. Im Unterschied zur Dichtung hat die Musik selbst jedoch keinen >belehrenden Nutzen< (>utilitas doctrinalis $<$ ), sondern dient nur dazu, die Menschen in die verschiedensten Stimmungen und Gefühle zu versetzen, aus denen heraus sie dann das tun und lernen, was ihnen die Dichtung vermittelt. Deswegen bedient man sich in der Kirche der Art von Gesang, die zur Frömmigkeit und Hingabe zwingt, im Gefecht der Pauken und Trompeten, um Zorn, Mut und Hoffnung auf den Sieg zu erregen und von der drohenden Todesgefahr abzulenken, bei Hochzeiten der Klänge, die Fröhlichkeit erregen. ${ }^{33}$

Das Abhängigkeitsverhältnis von >spiritus und Rhythmus ist auch die Ursache dafür, daß Campanella die Rückführung der menschlichen Musik auf eine ursprüngliche Sphärenharmonie, wie er sie Platon und Pythagoras zuschreibt, strikt ablehnt. Wie die Ziegen Ginster fressen und keinen Honig mögen, und also nicht jedem alles gleichermaßen bitter und süß ist, so würde auch die Musik der Sphären und der Engel, wenn es sie gibt, von einer gänzlich anderen Art sein und aus anderen Konsonanzen bestehen als die menschliche Musik. Die Magie, die Musik und Dichtung ausüben können, besteht also nicht in einer Angleichung an die Sphärenharmonie, wie es die neuplatonische Tradition will, sondern beruht ganz schlicht auf dem Vergnügen, das aus Metrum und Rhythmus entspringt.

Deswegen ist es offensichtlich, daß das Metrum Freude bereitet, weil es den spiritus nach seinem Rhythmus bewegt. Dies aber tut es nicht, weil es äußerliche Dinge nachahmt - das tut es nur akzidentiell -, sondern weil der spiritus selbst den Rhythmus nachahmt und ihn bewahrt. Denn das Ähnliche wird von Ähnlichem bewahrt. In diesem Vergnügen verbirgt der Dichter, wie in einem Köder, seine Sachkenntnis (sapientia), die gleichsam den Haken darstellt, mit dem er die Seelen einfängt, die sich gegen die Tugend sträuben. ${ }^{34}$

32 Ebd., S. 241: »Poëticae magiae vim esse in attrahendo eos ad audiendum, qui nolunt audire, et in docendo eos, qui ob ruditatem nequeunt aut taedio absterrentur [...].« Zum folgenden vgl. dort S. 241f.

33 Vgl. ebd., S. 221.

34 Ebd., S. 230: »Quas ob res liquet metrum delectationem habere, quoniam spiritum ad sui mensuram movet, non quia imitatur res exteriores, nisi per accidens, sed quia ipsius spiritus mensuram imitatur et servat: simile enim a simili servatur. Sub hac voluptate, tanquam sub esca, abscondit poëta sapientiam, quasi hamum, quo capit renitentes virtutibus animos.« 


\section{Kritik des Enthusiasmus}

Der rationalistischen Ausrichtung von Campanellas Poetik, wie sie sich in der Bestimmung des >exemplum< als logischer Form der Dichtung und der physiologischen Wirkung des Metrums ausdrückt, entspricht die Tatsache, daß Campanella jede Art von göttlicher Inspiration des Dichters, wie sie dem Modell des Enthusiasmus oder >furor poeticus< zugrundeliegt, strikt ablehnt. Was Campanella vom Dichter fordert, ist nicht göttliche Inspiration, sondern $>$ potentia $<$, >sapientia $<$ und $>$ probitas $<$. Unter $>$ probitas (Rechtschaffenheit) versteht Campanella die moralische Qualifikation des Dichters, das heißt, daß er die Frömmigkeit, die Gesetze und das moralisch richtige Verhalten liebt, daß er die Menschen besser machen will, die Tugend lobt und das Laster tadelt. Unter >sapientia< (Sachverstand) versteht Campanella die Forderung, daß der Dichter mit allen wichtigen Wissenschaften (Physiologie, Ethik, Politik, Geographie, Theologie usw.) bekannt ist und sachgemäß über die Dinge schreiben kann, die er behandelt. Unter dem Titel >potentia< behandelt Campanella schließlich die natürlichen Anlagen, die der Dichter mitbringen muß, nämlich Begabung und die Fähigkeit, die Verhaltensweisen der Menschen nachzuahmen und abzubilden. ${ }^{35}$

Die Behauptung, daß Dichter geboren und nicht gemacht werden, versteht Campanella wörtlich, indem er die Frage beantwortet, welche astrologische Konstellation für die Geburt eines Dichters besonders vorteilhaft ist. Campanella erkennt die Macht der Sterne allerdings nur insofern an, als die Sterne einen Hinweis auf die Verhaltensweisen und die Fähigkeiten liefern und die vier Säfte, die den Menschen beherrschen (schwarze und gelbe Galle, Phlegma und Blut), in ein bestimmtes Mischungsverhältnis setzen. Dichter können zu Melancholie und Einsamkeit neigen, daraus ergibt sich aber keine Vorherbestimmung, denn es ist die Seele, die allem, was aus dem Mischungsverhältnis entsteht, zustimmen muß. Während die Sterne deshalb nur >dispositiv < auf eine bestimmte Anlage hinweisen, können Engel und Dämonen viel direkteren, >persuasiven< Einfluß ausüben, nämlich indem sie auf das Gleichmaß der Säfte wirken, das Vorstellungsvermögen verändern und damit eine falsche Wahrnehmung (Wahnsinn, Verrücktheit, sfuror $<$ induzieren. Gott allein dagegen kommt ein physischer und unmittelbar wirkender (`physice et motive $<$ ) Einfluß $\mathrm{zu}^{36}$

35 Vgl. ebd., S. $267 f$.

36 Ebd., S. 268-270. 
Der Melancholie-Theorie des pseudo-aristotelischen >Problems 30.1<, die eine melancholische Disposition der besonders Begabten behauptet, bestreitet Campanella jede Gültigkeit. Bei den Schwarzgalligen, so Campanellas Argument, behindere der durch die Verbrennung der Galle entstehende $>$ Ruß < geradezu die Tätigkeit des Nachdenkens. Wenn wir die Kraft der Prophetie und der Dichtung haben, so deshalb, weil äußere Intelligenzen, gute oder böse, unseren Geist wie ein Instrument benutzten und unsere Fähigkeiten vervielfachten. Vergil und Platon hätten völlig richtig beobachtet, daß sich die Pythia und die Sibyllen nicht an das erinnerten, was sie gesagt hätten; dies sei jedoch auf natürliche Art zu erklären, denn durch die Heftigkeit und Feinheit der von außen induzierten >spiritus « würden diese einander so schnell ablösen, daß keine >affectio< stattfinde und deshalb keine Erinnerung zurückbleibe. Auch die Tatsache, daß Dichter bisweilen völlig ungebildete Menschen sind, die dennoch in ihren Dichtungen über die gelehrtesten Themen sprechen können, ist durch die Einwirkung von Dämonen zu erklären. ${ }^{37}$

Drei Arten von Propheten und Dichtern unterscheidet Campanella: göttliche, teuflische und menschliche. Die ersten verstehen, was sie sagen, sind von Gott erleuchtet und prophezeien in nüchternem Zustand wie David; die zweiten verstehen nicht, was sie sagen, und sind entweder verrückt oder vom Teufel besessen wie etwa die antiken Sibyllen; die dritten aber sind von Natur aus begabt, Verse zu bilden und die Verhaltensweisen der Menschen abzubilden. $\mathrm{Zu}$ dieser dritten Art von Dichtern, die durch Fleiß und intellektuell rationale Anstrengung zu hervorragenden Dichtern werden können, gehören etwa Horaz, Ovid und Catull. ${ }^{38}$ Nicht eine wie auch immer geartete Inspiration ist das Prinzip ihres Schaffens, sondern die Anstrengung der >ratio<, einem exemplarischen Gehalt seine rhetorisch und metrisch gestaltete Form zu geben.

Dämonen können auch menschliche Gestalt annehmen und auf diese Art prophetische Kräfte vortäuschen, wie Hieronymus in seiner Polemik gegen Montanus zeigt. Einen wahrhaft von Gott inspirierten Propheten kann man daran erkennen, daß Gott, wenn er in den Geist des Propheten eindringt, diesen Geist nicht an seiner Tätigkeit hindert, ein böser Geist dagegen zuerst die physiologischen Grundlagen, nämlich die >spiritus und die Säfte (>humores $<$ ) angreift und dadurch das Bewußtsein des eigenen Tuns schwinden läßt. Daraus erklärt sich auch die Tatsache, daß die Tätigkeit der Dämonen bei Vollmond und bei Melancholikern so viel

37 Vgl. ebd., S. 270-273.

38 Vgl. ebd., S. 272. 
stärker ist, denn in beiden Fällen ist das Säftegleichgewicht gestört. Wie ein Verbrecher um so eher zu einem Verbrechen bereit ist, wenn er die Schuld jemand anderem geben kann, so ist auch ein Dämon bei Vollmond oder melancholischem Temperament um so leichter bereit, von einer Seele Besitz zu ergreifen. ${ }^{39}$

\section{Das >Problem 30.1< und seine theologischen Implikationen}

Die Enthusiasmus-Kritik Campanellas wird nur auf dem Hintergrund der breiten Diskussion verständlich, die seit dem Ende des 15. Jahrhunderts, ausgehend von Marsilio Ficino, um den Begriff des Enthusiasmus geführt worden ist. ${ }^{40}$ Innerhalb der Philosophie Ficinos und seiner Nachfolger vor allem Francesco Patrizi wäre hier zu nennen - nimmt die Lehre vom Enthusiasmus oder $>$ furor poeticus $<$, das heißt die Lehre von der göttlichen Beseeltheit des Dichters, eine Schlüsselstellung insofern ein, als der Enthusiasmus die Form der Erkenntnis ist, in der der Mensch eines göttlichen Wissens teilhaftig werden kann, das er auf dem Weg eines bloß lo-

39 Vgl. ebd., S. 272.

40 Die folgende Darstellung ist notwendigerweise stark verkürzend. Stellvertretend verweise ich auf die ausführliche Darstellung bei Brann: The Debate over the Origin of Genius during the Italian Renaissance (wie Anm. 1). Zu Ficino im besonderen vgl. dort S. 82-107. Zu Ficinos Enthusiasmus-Theorie vgl. außerdem Michael J. B. Allen: Icastes: Marsilio Ficino's Interpretation of Plato's Sophist. Five Studies and a Critical Edition with Translation. Berkeley u. a. 1989; Michael J. B. Allen: The Platonism of Marsilio Ficino. A Study of His Phaedrus Commentary, Its Sources and Genesis. Berkeley u. a. 1984 (Publications of the UCLA Center for Medieval and Renaissance Studies 21), S. 41-67; André Chastel: Marcel Ficin et l'art. Geneva-Lille 1954 (Travaux d'humanisme et renaissance 14), S. 129-135; Sebastiano Gentile: »In margine all'epistola De divino furore di Marsilio Ficino«, in: Rinascimento 23 (1983), S. 33-77; Greenfield: Humanist and Scholastic Poetics. 1250-1500 (wie Anm. 3), S. 230-236; Jean-Claude Margolin: »La notion de >raptus< chez Ficin et Bovelles«, in: Marsile Ficin ou Les Mysteres platoniciens, hg. v. Stephane Toussaint. Paris 2002 (Les cahiers de l'humanisme 2), S. 267-297; Ute Oehlig: Die philosophische Begründung der Kunst bei Ficino. Stuttgart 1992 (Beiträge zur Altertumskunde 23), S. 51-83; Anne Sheppard: »The Influence of Hermias on Marsilio Ficino's Doctrine of Inspiration«, in: Journal of the Warburg and Courtauld Institutes 43 (1980), S. 97-109; Christoph J. Steppich: Numine afflatur. Die Inspiration des Dichters im Denken der Renaissance. Wiesbaden 2002 (Gratia 39), S. 146-214; Gary Tomlinson: Music in Renaissance Magic. Towards a Historiography of Others. Chicago 1993, S. 170-183; Clemens Zintzen: »Die Inspiration des Dichters. Ein Brief Ficinos aus dem Jahre 1457 «, in: Pratum Saraviense. Festgabe für Peter Steinmetz, hg. v. Woldemar Görler u. Severin Koster. Stuttgart 1990 (Palingenesia 30), S. 189-203. 
gisch schlußfolgernden Denkens nicht erreichen könnte. Signum des Enthusiasmus ist es etwa, daß der Dichter, der einer solchen Entrückung teilhaftig geworden ist, später, wenn er wieder in seinen normalen $\mathrm{Zu}$ stand zurückgekehrt ist, seine Verse selbst nicht mehr versteht, oder daß er schlicht im bewußten Zustand über das Wissen nicht verfügt, dessen Ausdruck seine Verse sind. Die Werke des echten, vom >furor poeticus< entrückten Dichters sind deshalb unmittelbar von Gott inspiriert und als solche für Ficino Ausdruck einer stheologia poetica<, einer dichterischen Theologie. Diese dichterische Theologie steht als >prisca theologia auf dem Rang der biblischen Offenbarung und hebt die Tätigkeit des Dichters damit natürlich weit über jede bloß rationale Tätigkeit im Sinne von Logik oder Rhetorik hinaus. Der Dichter steht als >vates , als Seher, in engster Verwandtschaft zum Propheten.

Diesem Modell einer göttlichen Inspiration entspricht das melancholische Temperament als physiologische Voraussetzung auf Seiten des Dichters, wie Ficino es im Anschluß an das >Problem 30.1< entwickelt. ${ }^{41}$ Drei Ursachen nennt Ficino, die den Melancholiker besonders für den Enthusiasmus disponieren. Die natürliche Ursache ist die geistige Tätigkeit selbst, die Kontemplation voraussetzt und damit in einem wechselseitigen Abhängigkeitsverhältnis zur schwarzen Galle, der Ursache des melancholischen Charakters, steht. Die physiologische Ursache ist die Feinheit der >spiritus< eines Melancholikers, die wiederum eine besondere Disposition für den Enthusiasmus darstellt, denn es sind nach Ficinos Überzeugung genau die >spiritus < des Dichters, die im Enthusiasmus von Gott ergriffen werden. Die astrologische Ursache besteht in den trockenen und kalten Eigenschaften Saturns, der im Horoskop aller Dichter die entscheidende Rolle spielt. ${ }^{42}$

Dieses neuplatonische Modell des Dichters als eines Sehers, der durch sein melancholisches Temperament ausgezeichnet ist und dessen Verse Ausdruck einer göttlichen Offenbarung sind, im Zustand der Entrückung empfangen, hat sofort zu scharfer Kritik geführt, wobei hier an erster Stelle der schon erwähnte Apologeticus de ratione artis poeticae (1492) Giro-

${ }^{41}$ Zur Melancholie-Theorie der Frühen Neuzeit verweise ich stellvertretend auf Raymond Klibansky, Erwin Panofsky und Fritz Saxl: Saturn und Melancholie. Studien zur Geschichte der Naturphilosophie und Medizin, der Religion und der Kunst, übers. v. Christa Buschendorf. Frankfurt a. M. 1990 und Schleiner: Melancholy, Genius and Utopia (wie Anm. 1).

42 Vgl. Marsilio Ficino: Three Books on Life, a Critical Edition and Translation with Introduction and Notes by Carol V. Kaske and John R. Clark. Binghamton-New York 1989 (Medieval \& Renaissance Texts \& Studies 57), Kap. 4. 
lamo Savonarolas zu nennen wäre. ${ }^{43}$ Savonarola zieht zwischen Dichtung und Theologie einen scharfen Trennstrich. Während die Theologie auf göttliche Offenbarung zurückgeht und die höchste Form menschlichen Wissens darstellt, ist die Dichtung eine Teildisziplin der Logik, das heißt eine bloß technische Fertigkeit, die als solche einen äußerst geringen Rang in der Ordnung menschlichen Wissens einnimmt. In dieser Zuordnung schließt Campanella sich über hundert Jahre später noch Savonarola an.

Was die Kritik der dichterischen Inspiration insbesondere betrifft, hat Campanella mit seiner Dämonisierung des Enthusiasmus einen weiteren Vorläufer in Gianfrancesco Picos della Mirandola Neun Büchern über das Vorherwissen, gegen abergläubische Nichtigkeiten und zum Schutz der Wahrheit der Religion verfaßt (De rerum praenotione libri novem. Pro veritate religionis/ contra superstitiosas vanitates editi, 1506). ${ }^{44}$ Trotz mancher Unterschiede - so lehnt Pico die Existenz einer natürlichen Magie ab und erklärt jede Magie zu dämonischer und damit schwarzer Magie - ist bei Pico die Grundstruktur der Argumentation Campanellas bereits vorgeprägt, was unter anderem auch darauf zurückgeht, daß beide in ihrer Terminologie stark von Thomas von Aquin abhängig sind. ${ }^{45}$

Wahres Vorherwissen fließt aus der wahren Religion oder aus natürlichen Zeichen, heißt es im fünften Kapitel des ersten Buches von De rerum praenotione, alles andere Vorherwissen aus der Finsternis des Aberglaubens (>superstitio<). Während das wahre Vorherwissen unmittelbar von Gott stammt oder über die Engel vermittelt ist und das natürliche Vorherwissen auf die Kenntnis der Zeichen zurückgeht, an denen etwa der Arzt den Verlauf einer Krankheit erkennt, geht die abergläubische Weissagung (>divinatio<) auf das Wirken von Dämonen zurück. Das siebte Kapitel, das den einzelnen Formen der Divination gewidmet ist, eröffnet Pico mit einem kurzen Referat der platonischen Enthusiasmus-

43 Zu Savonarola vgl. oben Anm. 3.

44 Gianfrancesco Pico della Mirandola: De rerum praenotione libri novem. Pro veritate religionis/ contra superstitiosas vanitates editi. Straßburg 1506. Das Werk ist jedoch schon vor 1502 entstanden, vgl. Charles B. Schmitt: Gianfrancesco Pico della Mirandola (1469-1533) and His Critique of Aristotle. The Hague 1967 (International Archives of the History of Ideas 23), S. 192. Vgl. ebendort zu den beiden anderen Ausgaben in den Opera omnia Picos, Basel 1573 und 1601. Vgl. außerdem Walker: Spiritual and Demonic Magic (wie Anm. 1), S. 146-151.

45 Zur thomistischen Herkunft von Picos Begrifflichkeit vgl. Thomas von Aquin: Summa theologica, II.2 q. 90-95. 
Theorie, die er, ganz wie später Campanella, als dämonische Besessenheit deutet. $^{46}$

Pico zitiert die delphische Pythia, die ihre Orakel zu geben pflegte, während sie von Apollon, einem bösartigen Dämon, erfüllt und ihres Geistes entrückt war. Von demselben Apollon heißt es auch, daß er den Dichtern erscheine, die dann im >furor< weissagten. Nach ihrem Beispiel hätten die späteren Dichter behauptet, von einem >furor< erfüllt zu sein. Für diese Entrückung der Dichter versuche Aristoteles im >Problem 30.1< eine natürliche Erklärung zu geben, indem er sie, wie die Entrückung der Sibyllen und Bacchen, nicht auf Krankheit oder dämonische Besessenheit zurückführe, sondern auf ein Ungleichgewicht der Säfte (>naturalis intemperies $<$ ), das heißt auf ein melancholisches Temperament. All jenen, die glaubten, göttlich inspiriert zu sein, billige er ein solches zu. Dies hätte dazu geführt, daß je weiter etwa die Sibyllen von der >ratio< entfernt gewesen wären, desto mehr man sie für Seherinnen gehalten hätte. Dies aber ist für Pico die eigentliche Verrücktheit, solcherart dämonisch Besessenen Glauben zu schenken und sich damit ihnen und den Dämonen auf Gedeih und Verderben auszuliefern. ${ }^{47}$ Schärfer kann man die Kritik an der neuplatonischen Sakralisierung des Enthusiasmus, wie sie sich bei Ficino findet, wohl kaum formulieren.

Sowohl für Pico wie für Campanella ist das >Problem 30.1< und das naturphilosophische Erklärungsmodell, das es für jede Art von Besessenheit, Inspiration und Prophetie zur Verfügung stellt, ein Ärgernis. Die Möglichkeit, daß es eine natürliche, das heißt nicht unmittelbar oder mittelbar (über Engel oder Dämonen) auf Gott zurückzuführende prophetische Begabung gibt, muß als eine Bedrohung des alleinigen Wahrheitsanspruches der biblischen Offenbarung verstanden werden. Genau dieses theologiekritische Potential des >Problems 30.1< hat Pietro Pomponazzi in seiner Abhandlung Über die Ursachen natürlicher Wirkungen, oder: Über Zauberei (De naturalium effectuum causis sive de incantationibus, 1520) zur Entfaltung gebracht. ${ }^{48}$ Er ist damit der eigentliche Gegner von Campanel-

46 Vgl. Pico: De rerum praenotione (wie Anm. 44), f. G4v f.

47 Vgl. ebd., f. H6r.

48 Pietro Pomponazzi: De naturalium effectuum causis sive de Incantationibus. Basel 1567. Ndr. Hildesheim-New York 1970. Pomponazzi selbst hat das Werk zu Lebzeiten nicht veröffentlicht, es wurde erst 1556 (zweite Aufl. 1567) von einem protestantischen Auswanderer in Basel herausgegeben. Die französische Teilübersetzung von Busson: Pietro Pomponazzi: Les causes des merveilles de la nature ou les enchantements. Paris 1930, war mir nicht zugänglich. $\mathrm{Zu}$ Pomponazzi vgl. stellvertretend Brann: The Debate over the Origin of Genius during the Italian Renaissance (wie 
la. Dieser macht ihn - zusammen mit Machiavelli - für nichts geringeres als den grassierenden Atheismus und die lutherischen und calvinistischen Häresien verantwortlich. ${ }^{49}$

Pomponazzis Werk ist der Versuch, für alle Arten von Phänomenen, die herkömmlicherweise dem Wirken von Engeln und Dämonen zugeschrieben wurden, naturphilosophische Erklärungen zu finden. ${ }^{50}$ Pomponazzi distanziert sich dabei ausdrücklich von denjenigen, die übernatürliche Phänomene ganz leugnen und sie etwa auf Betrug und Täuschung von Seiten der Priester zurückführen. Man müsse davon ausgehen, daß die gewichtigen und autoritativen Zeugnisse, die solche Phänomene bestätigen, tatsächlich wahr sind und sich deshalb gerade fragen, welche natürliche Erklärung diese Phänomene auf der Grundlage der aristotelischen Naturphilosophie finden könnten.

Eines dieser Phänomene ist die Tatsache, daß es Menschen gibt, die, ohne dabei auf ein technisch erworbenes Wissen, sei es mathematischastrologischer, sei es poetologischer oder sprachlicher Natur, zurückzugreifen, Zukünftiges vorhersagen oder große Dichtungen verfassen können, wie Platon in verschiedenen Dialogen und Aristoteles im $>$ Problem $30.1<$ behaupteten. $^{51}$ Pomponazzis komplexe naturphilosophische Erklärung kann hier nicht dargestellt werden, aber ihr Ergebnis lautet, daß solche Phänomene tatsächlich allein aus der >dispositio< der Gestirne als

Anm. 1), S. 167-177; Henri Busson: Les sources et le développement du rationalisme dans la littérature française de la Renaissance. Paris 1922; Hathaway: The Age of Criticism (wie Anm. 22), S. 329-340; Paul Oskar Kristeller: Acht Philosophen der italienischen Renaissance. Petrarca, Valla, Ficino, Pico, Pomponazzi, Telesio, Patrizi, Bruno, übersetzt von Elisabeth Blum. Weinheim 1986, S. 63-78; ders.: Aristotelismo e sincretismo nel pensiero di Pietro Pomponazzi. Padua 1988 (Saggi e testi 19); Martin L. Pine: Pietro Pomponazzi. Radical Philosopher of the Renaissance. Padua 1986 (Saggi e Testi 21), vor allem S. 235-274 (ältere Forschungsliteratur dort S. 239, Anm. 8); Antonino Poppi: »Fate, Fortune, Providence and Human Freedom«, in: The Cambridge History of Renaissance Philosophy, hg. v. Charles B. Schmitt u. a. Cambridge u. a. 1988, S. 641-667, hier S. 653-660; Walker: Spiritual and Demonic Magic (wie Anm. 1), S. 107-111; Eric Weil: »Die Philosophie des Pietro Pomponazzi«, in: Archiv für Geschichte der Philosophie 41 (1932), S. 127-176; Giancarlo Zanier: Richerche sulla diffusione e fortuna del De incantationibus di Pomponazzi. Firenze 1975 (Pubblicazioni del >Centro di Studi del pensiero filosofico del Cinquecento e del Seicento in Relazione ai problemi della scienza< del Consiglio nazionale delle ricerche. Serie I. Studi. 4).

49 Vgl. Brann: The Debate over the Origin of Genius during the Italian Renaissance (wie Anm. 1), S. 415f.

50 Vgl. zum folgenden Pomponazzi: De incantationibus (wie Anm. 48), S. 110-140.

51 Vgl. ebd., S. 124-131. 
Wirkursache und dem melancholischen Temperament als materialer Ursache erklärt werden können. Die Gestirne bestimmen das Temperament eines Menschen, dieses Temperament bildet dann den Charakter und die Begabungen aus.

Pomponazzi leugnet also nicht, daß Propheten und Dichter von einem >furor ergriffen werden und in Ekstase verfallen. Aber er erklärt diese Ekstase - und darin besteht der Unterschied zu Ficino $-{ }^{52}$ nicht als eine göttliche oder dämonische Besessenheit, sondern als eine physiologische Disposition, ein vom Verhältnis der Säfte bestimmtes Temperament, seinerseits auf astronomischen Einfluß zurückzuführen. Wo Ficino außerweltliche Kräfte für die Inspiration verantwortlich macht, da reduziert Pomponazzi die Inspiration auf ein medizinisches Phänomen. Dieses Erklärungsmodell hat erhebliche theologische Brisanz, denn damit ist es implizit möglich, nicht nur den Enthusiasmus des Dichters auf natürliche Art zu erklären, sondern tendenziell auch die Art von prophetischer Entrückung, wie sie in der Bibel berichtet wird. Wenn diese prophetischen Voraussagen nicht auf direktes göttliches Wirken zurückgehen müssen, sondern auch naturphilosophisch erklärt werden können, verliert die christliche Offenbarung erheblich an Beweiskraft. Es läßt sich aus ihr nicht mehr auf einen direkten Eingriff Gottes in die Geschehnisse der Welt rückschließen.

Dazu kommt zweitens die Tatsache, daß der naturphilosophische, physiologische Erklärungsansatz des >Problems 30.1< ausdrücklich die >ingeniöse $<$, prophetische Melancholie mit der krankhaften Melancholie, die für den herkömmlichen Wahnsinn verantwortlich ist, parallelisiert. Die physiologische Erklärung der Prophetie impliziert ihre Nähe zum Wahnsinn, denn beide, Prophetie und Wahnsinn, werden damit auf ein bestimmtes Verhältnis der Säfte zurückgeführt. Eine solche Parallele wird von der theologischen Erklärung der Prophetie als mittelbares oder unmittelbares Wirken Gottes natürlich ausgeschlossen. Diese Parallele hat weitreichende Konsequenzen, denn damit besteht bei jedem, der Stimmen hört oder Gesichte hat, die Möglichkeit, daß es sich nicht um einen Propheten, sondern nur um einen Verrückten handelt. Religiöse Erscheinungen können in einem medizinischen Sinne pathologisiert werden.

Vor dem Hintergrund dieser beiden Implikationen von Pomponazzis De incantationibus ist es kaum verwunderlich, daß Pomponazzi sein Werk selbst nicht veröffentlicht hat und daß es, als es 1556 posthum gedruckt wurde, auf den päpstlichen Index der verbotenen Bücher kam. Diese bei-

52 So bereits Walker: Spiritual and Demonic Magic (wie Anm. 1), S. 110. 
den Implikationen der Melancholie-Theorie des >Problems 30.1< dürften deshalb auch die Ursache dafür sein, daß sowohl Pico wie Campanella diesem Erklärungsmodell jede Gültigkeit bestreiten.

\section{Schlußfolgerungen}

Im Vergleich zu den skizzierten theologischen Implikationen der Melancholie-Theorie sind die poetologischen Konsequenzen, wie sie sich paradigmatisch an der Poetica Campanellas ablesen lassen, relativ unbedeutend. Für die Dichtungstheorie wird die Berufung auf göttliche Inspiration schwierig, denn entweder der Dichter tritt damit neben den Propheten, wie es der Neuplatonismus Ficinos impliziert, und das heißt, mit der Berufung auf den Enthusiasmus wird auch ein äußerst prätentiöser theologischer Anspruch erhoben. Oder aber die Inspiration wird naturphilosophisch erklärt. Dann aber tritt der Dichter neben den Verrückten, wie es Pomponazzis Deutung der Melancholie-Theorie impliziert. Solange die Dichtungstheorie nicht über einen Genie-Begriff verfügt, mit dem sich die Nähe zum Verrückten ins Positive umdeuten läßt, ist eine solche Nähe mit der Legitimation der Dichtung schwer vereinbar.

Deswegen entscheidet sich das 17. Jahrhundert, mit Campanella, für eine rationalistische Bestimmung der Dichtung als ein argumentatives und damit vernunftgesteuertes, lehr- und lernbares Verfahren, das einer wie auch immer gearteten Inspiration gar nicht bedarf. In der Konsequenz kann dann, wie Gianfrancesco Pico es vorgezeichnet hat, jede nicht theologisch beglaubigte Inspiration für dämonische Besessenheit erklärt werden, ohne die Dichtung in ihrer Legitimation zu beschädigen. Die Dichtung wird von der theologischen Inspirationsvorstellung abgekoppelt und allein auf den weltlichen Bereich beschränkt. Sie wird ausschließlich zu einem technischen Verfahren, das durch exemplarischen Gehalt und metrische Form der Besserung menschlichen Verhaltens dient. Mit dieser Lösung, so meine Ausgangsthese, ist Campanella richtungsweisend bis hin zur aufkommenden Genie-Bewegung in der Mitte des 18. Jahrhunderts. 